\title{
Paresthesiae During Radiofrequency NeURolysis of LUMbar SYMPATHETIC TRUNK IN CRPS: HumAN EVIDENCE OF A SYMPATHO- SEnsory CONNECTION?
}

\begin{abstract}
Abdallah I. Kabbara, MD, Thomas Chelimsky, MD, and Mark V. Boswell, MD, PhD
Radiofrequency heat lesioning has been advocated to prolong the duration of therapeutic effect of lumbar sympathetic block in Complex Region Pain Syndrome (CRPS) of the lower extremity. Prior to radiofrequency lesioning of the lumbar sympathetic trunk, sensory and motor stimulation may be used to verify that the active needle tip is not adjacent to a spinal nerve to avoid unwanted neural injury. However, the value of sensory stimulation to aid in precise needle positioning at the desired target remains controversial. In this report we describe our observations using sensory stimulation to aid in needle placement for radiofrequency lesioning. We

confirm a novel observation that paresthesiae to the lumbar sympathetic trunk is possible and hypothesize on pathophysiologic meaning.

Two representative patients are presented who underwent sensory stimulation of the lumbar sympathetic trunk during needle placement for lumbar sympathetic radiofrequency neurolysis. Sensory stimulation was elicited at the L3 or L4 vertebral level using a $15 \mathrm{~cm}$ insulated radiofrequency needle with a 5 $\mathrm{mm}$ active tip. During sensory stimulation, patients described paresthesiae to the ipsilateral buttock and lower extremity. Stimulation intensity was less
\end{abstract}

Lumbar sympathetic block with local anesthetic is a diagnostic and a therapeutic tool for treatment of complex region pain syndrome (CRPS) in the lower extremity. Radiofrequency heat lesioning (radiofrequency) of the lumbar sympathetic trunk has been advocated to prolong the duration of therapeutic effect (1). Radiofrequency of the sympathetic trunk or chain also has been used to provide sympathectomy to improve blood flow in the lower extremity in peripheral vascular disease (2). Other uses of radiofrequency include treating sacroiliac joint pain and pain associated with obturator and femoral nerve neuralgias $(3,4)$. More recently, pulsed radiofrequency has sion radiofrequency $(5,6)$. Sensory and motor stimulation may be used to verify that the needle tip is not adjacent to a spi-

From Departments of Anesthesiology and neurology, Case Western Reserve University School of Medicine and University Hospitals of Cleveland, Cleveland, Ohio. Address Correspondence: Mark V. Boswell, MD, PhD, Department of Anesthesiology, land, Ohio-44106.Email: mark.boswell@uhhs.com Funding: There was no external funding in preparation of this manuscript. been proposed as an alternative to heat leUniversity Hospitals, 11100 Euclid Avenue, Cleve- nal nerve to avoid unwanted neural injury (7). However, the value of sensory stimulation to aid in precise needle positioning at the desired target remains controversial $(8,9)$. We have found that sensory stimulation is useful in needle placement, much like sensory stimulation for placement of spinal cord stimulator electrodes. That is, paresthesiae may help guide proper needle placement. Standard heat lesion radiofrequency is not generally advocated for peripheral nerve ablation, but pulsed radiofrequency appears useful for treating peripheral neuralgias, without the risk of post-procedure neuritis or deafferentation pain.

During needle placement for radiofrequency neurolysis, we noted that sensory stimulation of the sympathetic trunk at the L3 or L4 levels produced ipsilateral paresthesiae to the buttock and lower extremity, an observation previously reported by van Kleef and Sluitjer (10) for stimulation of the communicating ramus of the sympathetic trunk at the lumbar vertebral level. We use sensory stimulation routinely during placement of radiofrequency needles for lumbar radiofrequency sympatholysis, to avoid positioning the active tip adjacent to a spinal nerve and than $0.8 \mathrm{~V}$ at $50 \mathrm{~Hz}$ at impedance of 300 Ohms or less. Motor stimulation did not occur up to the maximum voltage tested (2.0 V at $2 \mathrm{~Hz}$ )

Sensory stimulation of the lumbar sympathetic trunk may be used to aid in localization of the active tip of the radiofrequency needle, in preparation for lesioning. A dermatomal sensory pattern was observed, suggesting that afferent sensory fibers travel in the lumbar sympathetic trunk. The implications of this observation for understanding the mechanism of CRPS-related pain are discussed.

Keywords: CRPS, radiofrequency, sensory stimulation, sympathetic trunk to precisely localize the needle at the sympathetic chain. In this report we describe two representative cases where pulsed radiofrequency was employed for pain control, using sensory stimulation of the sympathetic chain to precisely guide needle positioning. In both cases, stimulation of the lumbar sympathetic trunk produced ipsilateral paresthesiae in a dermatomallike pattern to the back and lower extremity. This referral pattern may support the understanding of mechanisms of CRPS related pain based on animal models or suggests previously unrecognized neuroanatomic connections.

\section{Description/Methods}

A 44-year-old female with a history of right lower extremity CRPS of 2 years' duration related to an ankle sprain that improved temporarily with local anesthetic lumbar sympathetic block. A 22 gauge 15 $\mathrm{cm}$ insulated radiofrequency needle with a $5 \mathrm{~mm}$ active tip (Sluijter-Mehta Kit [SMK]; Radionics, Tyco Healthcare, Burlington, MA.) was positioned at the right anterior-lateral aspect of L4, just anterior to the fascia of the psoas muscle, the presumed location of the lumbar sympathetic trunk. Fluoroscopic guidance was used 


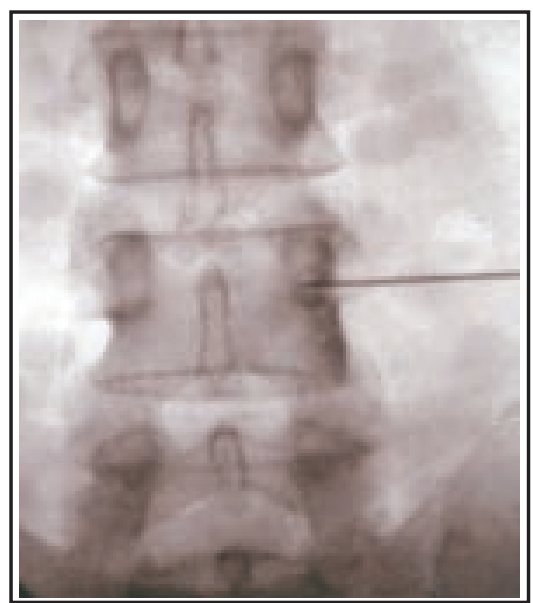

Fig. 1. Anterior-posterior view (right is to the right of the image) of insulated radiofrequency needle in position at the $L 4$ vertebral body for stimulation of the lumbar sympathetic chain. Stimulation at $0.7 \mathrm{~V}$ produced paresthesiae to the right posterior calf and ankle. The needle tip was not near spinal nerve or lumbar plexus.

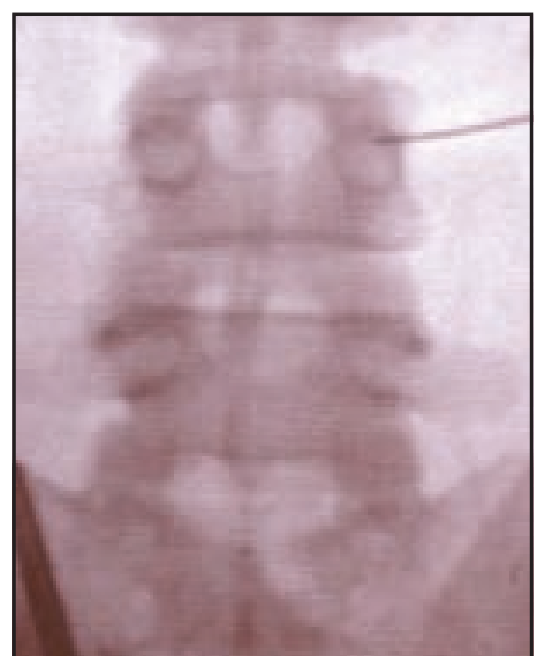

Fig. 4. APview after slight readjustment of needle position producing sensory stimulation at $0.3 \mathrm{~V}$.

for needle placement. Iohexol injection demonstrated typical spread at the anterolateral aspect of the vertebral body of L4. The needle tip was not near the intervertebral foramen or spinal nerve (Figs. 1 and 2). Needle position was adjusted until maximal sensitivity was obtained with electrical stimulation. A Radionics Plus radiofrequency Generator (RFG-3C ${ }^{\mathrm{TM}}$ Plus; Radionics, Burlington, MA.) was used for stimulation and lesioning. At a stimulation frequency of $50 \mathrm{~Hz}$ (imped-

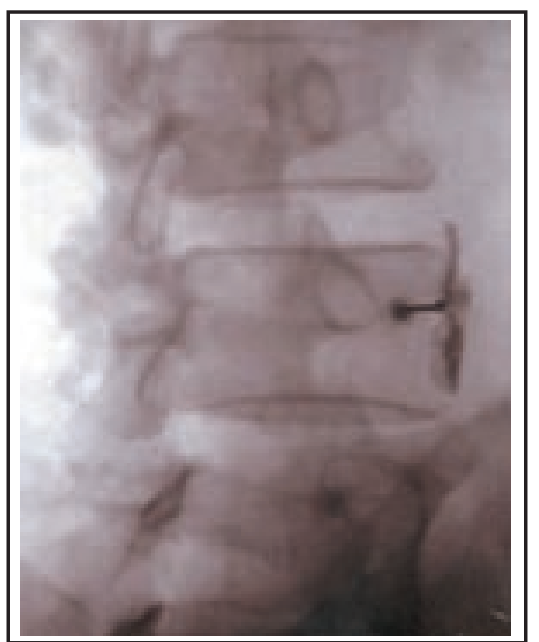

Fig. 2. Lateral view of radiofrequency needle in position at L4 for lumbar sympathetic block, demonstrating adequate distance of active tip from the spinal nerve.

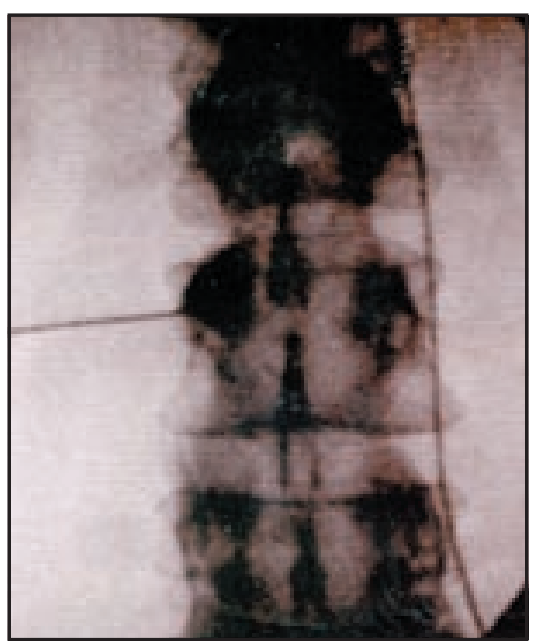

Fig. 5. Anterior-posterior view (left is to the left of the image) of insulated radiofrequency needle in position at the L3 vertebral body. Stimulation at $0.3 \mathrm{~V}$ produced paresthesiae to the patient's left anterolateral thigh. The active tip was not adjacent to spinal nerve or lumbar plexus.

ance of $300 \mathrm{Ohms}$ ) the patient reported paresthesiae to the right posterior calf and ankle, at a threshold of $0.7 \mathrm{~V}$. Motor stimulation was negative up to the maximum tested voltage of $2.0 \mathrm{~V}$. Three pulsed radiofrequency "lesions" were generated at $\mathrm{L} 4$, each at a frequency of $2 \mathrm{~Hz}$, with a 20 ms pulse width for $120 \mathrm{sec}$ at $42^{\circ} \mathrm{C}$. The radiofrequency treatment provided more

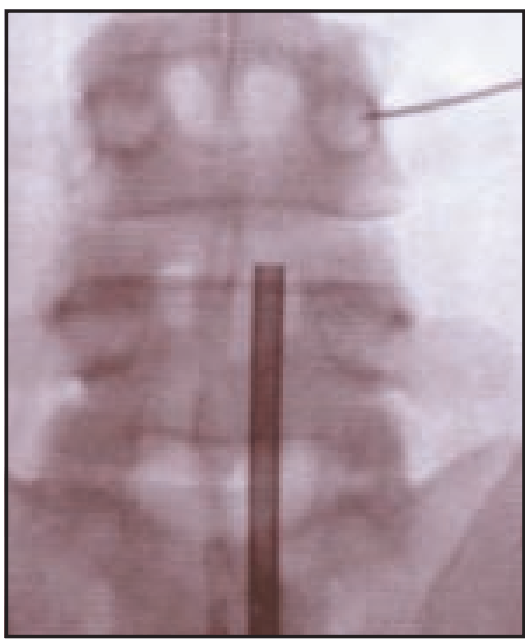

Fig. 3. AP view with no sensory stimulation elicited.

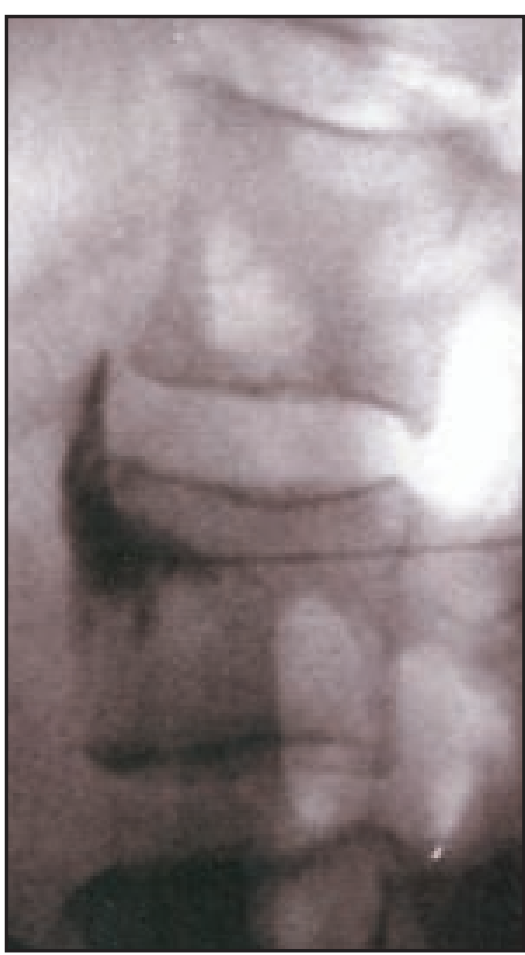

Fig. 6. Lateral view of radiofrequency needle in position for radiofrequency of lumbar sympathetic chain at L3, demonstrating adequate distance from the spinal nerve.

than $50 \%$ pain relief for 3 months. Movement of the active tip 2 or $3 \mathrm{~mm}$ from target eliminated stimulation-induced paresthesiae (Figs. 3 and 4, a different patient). This suggests that paresthesiae were elicited from sympathetic trunk activation, rather than nonspecific stimulation of the 
periosteum or adjacent non-neural tissue. A 42-year-old female with a 3-year history of CRPS in the left lower extremity after Achilles tendon injury, noted temporary pain relief with local anesthetic lumbar sympathetic block. The tip of an SMK needle was advanced to the left lumbar sympathetic trunk at L3, using fluoroscopic visualization. Injection of Iohexol contrast demonstrated typical spread at the anterolateral aspect of the vertebral body of L3 (Figs 5 and 6). Precise needle positioning was guided by sensory stimulation. Sensory stimulation of the lumbar sympathetic trunk at $50 \mathrm{~Hz}$ produced distinct paresthesiae to the patient's left anterolateral thigh, at a threshold of $0.3 \mathrm{~V}$ (impedance of $300 \mathrm{ohms}$ ). Motor stimulation was negative at $2.0 \mathrm{~V}$. The active tip was not adjacent to a lumbar nerve root or the intervertebral foramen. Three pulsed radiofrequency lesions were delivered at 2 $\mathrm{Hz}, 20 \mathrm{~ms}$ duration for $120 \mathrm{sec}$. The patient obtained more than 50\% pain relief for 3 months.

\section{DisCUSSION}

Lumbar sympathetic block with local anesthetic has been used for years to produce sympathectomy of the lower extremity for treatment of CRPS. More recently, radiofrequency has been used in an attempt to prolong the duration of sympathectomy. Haynsworth (11) reported that radiofrequency ablation of the sympathetic chain provided longer duration of pain relief and fewer complications than phenol neurolysis.

van Kleef and Sluitjer (10) have reported sensory stimulation of the rami communicantes of the sympathetic trunk and found it helpful from a technical standpoint, because paresthesiae allowed accurate positioning of the needle tip for radiofrequency lesioning. However, they did not comment on the implications of their observation. We have documented that sensory stimulation with the radiofrequency needle after placement in the traditional position required for lumbar sympathetic block reliably produces paresthesiae to the ipsilateral low back and proximal lower extremity. Paresthesiae in a dermatomal pattern for the level stimulated were elicited. Needle position was documented fluoroscopically, and the active tip of the insulated needle was not adjacent to a nerve root, lumbar plexus or nerve. With the active tip of the insulated needle in the anatomical position of the sympathetic chain, anterior to the fascia of the psoas muscle, paresthesiae were readily obtainable. Repositioning the active tip several $\mathrm{mm}$ away from the point of maximum stimulation reliably abolished the paresthesiae. This indicated that paresthesiae elicited by stimulation were specific for the sympathetic trunk or associated ganglion and not due to stimulation of periosteum or adjacent non-neural tissue.

Our observations may be useful when considering the pathophysiology of chronic pain states, such as sympathetically maintained pain and CRPS. Abnormal sympathetic connections have been demonstrated in dorsal root ganglia after injury (12). Some studies suggest an important role of noradrenaline released by the sympathetic postganglionic axons in pain generation. This assumes efferent activity of the sympathetic nervous system in the pain process. By activating alphaadrenoreceptors, noradrenaline stimulates primary afferent neurons present in the injured tissue and may cause pain (13). Such connections may involve sympathetic synapses in the dorsal root ganglia DRG $(13,14)$. The concept of sprouting of sympathetic fibers in the dorsal root ganglion has been attributed to Raymon y Cajal (15).

Our observations suggest three hypotheses: (I) the electrical stimulus excites sympathetic nerve fibers which have a pathologic synapse upon dorsal root ganglion cells These, in turn, are responsible for the patient's sensory experience. This hypothesis would constitute human evidence of the lumbar sympathetic -dorsal root ganglia connection demonstrated in rats (13) (II) the sympathetic ganglion contains nerve fibers which conduct antidromically and synapse on dorsal horn cells in the spinal cord which then produce the sensation felt by the patient. (III) Afferent fibers actually travel through the sympathetic ganglion and are being stimulated by the radiofrequency needle directly. Evidence for the first hypothesis only derives from animal models in CRPS (13). Since both patients presented had CRPS, it would be of interest to know whether non-CRPS patients report a similar experience. If no sensation is reported by non -CRPS patients, then the first hypothesis would be most likely and our case report may provide the first known human evidence of the hypothesized connection between sympathetic and dorsal root ganglia in CRPS. If non-CRPS pa- tients also report the sensation then one of the two anatomic explanations may be more likely and would need further exploration.

If afferent sensory fibers are present in the sympathetic trunk this might provides a novel explanation for how local anesthetic blockade can produce pain relief in CRPS. Rather than blocking presumed efferent sympathetic activity and thereby reducing peripheral sensitization, local anesthetic blockade may also inhibit painful afferent neural conduction directly in the sympathetic trunk. Although pulsed radiofrequency is not yet widely recognized as a treatment modality, pulsed radiofrequency of the DRG has been shown to result in expression of cFOS in the dorsal horn in the rat (14).

In conclusion, we find that eliciting sensory paresthesiae for precise localization of the active tip of the radiofrequency needle is useful in preparation for lesioning of the sympathetic trunk. Our observations warrant further study. Studies of patients without CRPS, documenting sensory stimulation patterns, including measuring latency times and sensory voltage thresholds, may help confirm the presence of afferent sensory fibers in the sympathetic chain. Additionally, it would be useful to correlate skin blood flow changes with the distribution of paraesthesiae in-patients undergoing radiofrequency of the lumbar sympathetic chain for treatment of CRPS.

Author Affiliation:
Abdallah I. Kabbara, MD
Fellow in Pain Medicine
Department of Anesthesiology
University Hospitals of Cleveland
Cleveland, Ohio 44106
Thomas Chelimsky, MD
Associate Professor of Neurology
University Hospitals of Cleveland
Cleveland, Ohio 44106
Mark V. Boswell, MD, PhD
Associate Professor of Anesthesiology
Chief, Division of Pain Medicine
Department of Anesthesiology
Director, Pain Medicine Fellowship
Program
Case Western Reserve University
School of Medicine and University
Hospitals of Cleveland
11100 Euclid Avenue
Cleveland, Ohio-44106
E-mail: mark.boswell@uhhs.com




\section{References}

1. Rocco AG. Radiofrequency lumbar sympatholysis. The evolution of a technique for managing sympathetically maintained pain. Reg Anesth. 1995; 20:3-12.

2. Dominkus $M$, Kepplinger B, Bauer $W$ et al Percutaneous radiofrequency thermolesion of the sympathetic chain in the treatment of peripheral vascular disease. Acta Med Austriaca 1991; 18:S69-S70.

3. Rathmell JP, Tarver JM, Memon Z. Radiofrequency sacroiliac joint denervation: the devil is in the details. Reg Anesth Pain Med 2001; 26: 592-593.

4. Kawaguchi M, Hashizume K, Iwata T et al. Percutaneous radiofrequency lesioning of sensory branches of the obturator and femoral nerves for the treatment of hip joint pain. Reg Anesth Pain Med 2001; 26: 576-581.

5. Sluijter ME. Radiofrequency. Part 1. A review of radiofrequency procedures in the lumbar region. Flivopress, Amsterdam, 2001.

6. Munglani R. The longer term effect of pulsed radiofrequency for neuropathic pain. Pain 1999; 80:437-439.

7. Lord SM, Barnsley L, Wallis BJ et al. Percutaneous radio-frequency neurotomy for chronic cervical zygapophyseal-joint pain. N Eng J Med 1996; 335:1721-1726.

8. Dreyfuss P, Halbrook B, Pauza K. Efficacy and validity of radiofrequency neurotomy for chronic lumbar zygapophysial joint pain. Spine 2000; 25:1270-1277.

9. Windsor RE. Radiofrequency lumbar zygapophysial (facet) joint denervation: $A$ preliminary report of a new concept. Pain Physician 2003; 6:119-123.

10. van Kleef M, Sluitjer ME. Radiofrequency lesions of the Dorsal Root Ganglion in the Treatment of Spinal Pain. Chapter 4. Treatment of Spinal Pain with Radiofrequency Techniques - Procedures. Universitaire Pers Maastricht, 1996, pp 44.
11. Haynsworth RF Jr, Noe CE. Percutaneous lum bar sympathectomy: A comparison of radiofrequency denervation versus phenol neurolysis. Anesthesiology 1991; 74:459-463.

12. Katz DM. Adler JE, Black IB. Catecholaminergic primary sensory neurons: Autonomic targets and mechanisms of transmitter regulation. Fed Proc 1987; 46:24-29.

13. McLachlan EM, Janig W, Devor M et al. Peripheral nerve injury triggers noradrenergic sprouting within dorsal root ganglia. Nature 1993; 363:543-546.

14. Higuchi Y, Nashold BS Jr, Sluijter $M$ et al. Exposure of the dorsal root ganglion in rats to pulsed radiofrequency currents activates dorsal horn lamina I and II neurons. Neurosurgery 2002; 50:850-855.

15. Garcia-Poblete E, Fernandez-Garcia $H$, Moro-Rodriguez $E$ et al. Sympathetic sprouting in dorsal root ganglia (DRG): A recent histological finding? Histol Histopathol 2003; 18:575-86. 